\title{
Elevación crónica de Creatincinasa
}

\author{
Pablo Franquelo Morales ${ }^{a}$, Mubarak Alramadam Alramadam ${ }^{b}$, \\ Enrique Prada De Medioc ${ }^{c}$ Félix González Martíneza
}

\begin{abstract}
a Servicio de Urgencias. Hospital Virgen de la Luz, Cuenca.

b Servicio de Endocrinología. Hospital Virgen de la Luz, Cuenca.

- Servicio de Análisis Clínicos. Hospital Virgen de la Luz, Cuenca.

\section{Correspondencia:}

Pablo Franquelo Morales, Servicio de Urgencias del Hospital Virgen de la Luz, C/ Hermandad de Donantes de Sangre $n^{\circ} 2$,

16002 - Cuenca, España.

E-mail: pfranquelo@yahoo.es.
\end{abstract}

Recibido el 8 mayo de 2009.

Aceptado para su publicación el 20 de agosto de 2009.

\section{RESUMEN}

Las miopatías son entidades en las que se produce afectación musculoesquelética con elevación de creatincinasa en suero. La Enfermedad de Addison y la Hipertermia Maligna constituyen causas poco comunes de miopatía. La Enfermedad de Addison corresponde a una insuficiencia suprarrenal primaria producida por un déficit de glucocorticoides, mineralocorticoides y andrógenos por destrucción de la corteza suprarrenal, cuyos síntomas son inespecíficos y el tratamiento es con hidrocortisona. La Hipertermia Maligna se produce por una alteración genética en la que los anestésicos inhalados y los relajantes musculares desencadenan una hipertermia progresiva, con rigidez muscular, taquicardia, hipotensión, rabdomiolisis, y coagulación intravascular diseminada.

Palabras clave. Enfermedad de Addison. Hipertermia Maligna. Creatincinasa.

\section{ABSTRACT}

\section{Chronic creatinine elevation}

Myopathy is a disease that affects the musculoskeletal system with elevated serum creatinine. Addison's Disease and Malignant Hyperthermia, are rare causes of myopathy. Addison's disease, also known as primary adrenal insufficiency, occurs when the adrenal gland does not produce enough glucocorticoids, mineralocorticoids and androgens because of the destruction of the adrenal cortex. Symptoms are non-specific and it is treated with hydrocortisone. Malignant Hyperthermia is a genetic disorder, in which inhaled anaesthetics and muscle relaxants, trigger progressive hyperthermia with muscle rigidity, tachycardia, hypotension, rhabdomyolysis, and disseminated intravascular coagulation.

Key words. Addison Disease, Malignant Hyperthermia, Creatine Kinase.

\section{INTRODUCCIÓN}

Las miopatías son entidades en las que se produce afectación de la fibra musculoesquelética, provocando una elevación de creatincinasa (CK) en suero. Se clasifican según su etiología en congénitas, inflamatorias, metabólicas, de membrana y distrofias musculares. Fisiopatológicamente constituyen la expresión de un aumento de la permeabilidad de la membrana celular del músculo como consecuencia de diversas situaciones clínicas. La Enfermedad de Addison (EA) y la Hipertermia maligna (HM) constituyen causas poco comunes de miopatía y, aunque todavía no se ha definido claramente el significado de la persistencia de la elevación de la CK en los pacientes con EA, la afectación musculoesquelética no es un hallazgo frecuente. A continuación presentamos el caso de un varón joven con elevación persistente de la CK sérica, cuyo hermano falleció por una hipertermia maligna.

\section{OBSERVACIONES CLÍNICAS}

Varón de 17 años sin alergias medicamentosas conocidas, amigdalectomizado sin incidencias, que consultó por epigastralgia y debilidad muscular generalizada más acusada en los miembros inferiores (MMII) desde hacía 6 meses. Refería una sensación de mareo, cansancio con la deglución y astenia en relación con ejercicios cada vez menos intensos. La clínica mejoraba con el descanso y en los últimos 2 meses había perdido $3 \mathrm{~kg}$ de peso. Realizaba ejercicio físico hasta hacía un año sin presentar calambres ni episodios de fatigabilidad marcada. Negaba disfagia, diplopía y 
sintomatología bulbar. No refería antecedentes de enfermedades neurodegenerativas, miocardiopatías, alteraciones motoras ni de consanguinidad familiares.

En la exploración física presentaba buen estado general con tensión arterial de 140/76 mm/ $\mathrm{Hg}$ y sin afectación de los pares craneales. Los reflejos osteotendinosos en MMII se encontraban disminuidos y presentaba dificultad para caminar de puntillas e imposibilidad para hacerlo de cuclillas, con la fuerza debilitada y una gran fatigabilidad ante ejercicios repetitivos.
Registró valores de CK superiores a $4.000 \mathrm{U} / \mathrm{L}$, por lo que establecimos un diagnóstico diferencial con las posibles causas de elevación de CK en suero (tabla 1).Tras su ingreso, la $\mathrm{CK}$ disminuyó, aunque persistió elevada (2.345 U/L), con una TSH de $4.19 \mathrm{mUI} / \mathrm{L}$, anticuerpos anti-21-hidroxilasa positivos, hormona adenocorticotropa (ACTH) de $1046 \mathrm{pg} / \mathrm{mL}$ y cortisol basal $<1 \mathrm{mgr} / \mathrm{dL}$. Los anticuerpos anti -TPO fueron negativos, las hormonas de autoinmunidad, incluyendo ANA, complemento, anticuerpo antimúsculo liso, antimitocondriales, antimúsculo cardiaco y ANCA, así como la paratohormona, no se vieron alterados.

\begin{tabular}{|c|c|}
\hline Causas cardíacas & $\begin{array}{l}\text { Infarto de miocardio } \\
\text { Insuficiencia cardiaca } \\
\text { Pericarditis } \\
\text { Arritmias } \\
\text { Insuficiencia coronaria } \\
\text { Miocarditis } \\
\text { Tromboembolismo pulmonar } \\
\text { Cardioversión eléctrica }\end{array}$ \\
\hline Causas farmacológicas y tóxicas & $\begin{array}{l}\text { Analgésicos } \\
\text { Insulina } \\
\text { Heparina } \\
\text { Meperidina } \\
\text { Estatinas } \\
\text { Barbitúricos } \\
\text { Diuréticos } \\
\text { Penicilinas } \\
\text { Ampicilina } \\
\text { Alcohol } \\
\text { Cocaína } \\
\text { Clofibratros } \\
\text { Clonidina } \\
\text { Fenotiazinas } \\
\text { Narcóticos } \\
\end{array}$ \\
\hline Causas endocrinas & $\begin{array}{l}\text { Hiper e hipotiroidismo } \\
\text { Enf. De Addison } \\
\text { Síndrome de Cushing }\end{array}$ \\
\hline Causas musculares & $\begin{array}{l}\text { Polimiositis } \\
\text { Distrofia muscular de Becker } \\
\text { Rabdiomiolisis } \\
\text { Enf. De Duchenne } \\
\text { Miositis } \\
\text { Enf. De Kugelburg-Welander }\end{array}$ \\
\hline Causas neoplásicas & $\begin{array}{l}\text { Carcinoma gástrico } \\
\text { Carcinoma de próstata }\end{array}$ \\
\hline Causas psiquiátricas & Esquizofrenia \\
\hline Otras & $\begin{array}{l}\text { Ejercicio físico } \\
\text { Sd. de Reye } \\
\text { Hipertermia maligna } \\
\text { Picadura de abeja } \\
\text { Recién nacido } \\
\text { Hemólisis } \\
\text { Síndrome neuroléptico maligno } \\
\text { Cirugías } \\
\text { Inyecciones IM y SC } \\
\text { Sd. Compartimental } \\
\text { Traumatismo muscular } \\
\text { Convulsiones }\end{array}$ \\
\hline
\end{tabular}

Tabla 1. Causas de elevación de CK en suero ${ }^{7-9}$. 
Autoinmune (70\%)

\section{Infecciones}

Asociadas a VIH/inmunosupresión

Metástasis

Enfermedades infiltrativas

Hemorragia o infarto suprarrenal

Fármacos
Aislada o formando parte de un síndrome poliglandular

(Enf. de Addison) autoinmune (SPA): SPA tipo I: Adrenalitis

autoinmune, Candidiasis mucocutánea crónica, Hipoparatiroidismo

SPA tipo II: Adrenalitis autoinmune, Enfermedad tiroidea autoinmune, Diabetes mellitus tipo 1

TBC, Sífilis, Micosis (Histoplasmosis, Blastomicosis)

CMV, Sífilis, IMAI, criptococo, toxoplasma, linfoma suprarrenal, sarcoma de Kaposi, etc.

Pulmón, Tiroides, Carcinomas o Linfomas gástricos, Melanoma, Riñón, Mama, Vejiga

Amiloidosis, Sarcoidosis ${ }^{10}$, Hemocromatosis

ketoconazol, rifampicina, fenitoína, fenobarbital, minoglutetimida, metopirona, mitotano, etomidato

Hiperplasia suprarrenal congénita, Adrenoleucodistrofia y

Adrenomieloneuropatía, Deficiencia familiar y resistencia a los

glucocorticoides, Trastornos de la coagulación, Hemorragia adrenal, Síndrome Antifosfolipídico, Sepsis

Tabla 2. Causas de insuficiencia suprarrenal primaria.

Realizamos radiografía de tórax, ecocardiograma y resonancia magnética de MMII y abdomen sin revelar patología. La prueba de Mantoux fue negativa y la espirometría reveló una disfunción ventilatoria restrictiva de grado leve (se cansaba mucho y no podía soplar).

El electromiograma demostró signos de polineuropatía mixta de carácter leve y el paciente desestimó la realización de una biopsia muscular. Ante la sospecha de una miopatía como origen del cuadro, se practicó Test de estimulación de ACTH sintética (Synacthen) ${ }^{1}$, objetivándose niveles de cortisol indetectables, lo que diagnosticó una insuficiencia suprarrenal primaria por Addison. Tras administrar hidroaltesona, mejoró notablemente, pudiendo caminar sin incidencias, no obstante, los valores de CK permanecían elevados (654 $\mathrm{U} / \mathrm{L}$ ). El fallecimiento de su hermano, por hipertermia maligna tras una anestesia por cirugía de criptorquidia, sugería una posible susceptibilidad genética como explicación de esos niveles de CK tan elevados, dato que nos orientó hacia una miopatía como explicación del cuadro.

\section{COMENTARIOS}

La EA corresponde a una insuficiencia suprarrenal primaria producida por un déficit de glucocorticoides, mineralocorticoides y andrógenos como consecuencia de una destrucción de la corteza suprarrenal superior al $90 \%$, lo cual conlleva un aumento compensador de ACTH. En la actualidad la Adrenalitis autoinmune es la causa más frecuente, junto a la Tuberculosis, que ha disminuido su incidencia representando un $20 \%$ de los $\operatorname{casos}^{2,3}$ (tabla 2).

La mayoría de los síntomas de la insuficiencia suprarrenal son inespecíficos y aparecen de forma aislada, por lo es fácil que pasen inadvertidos si no se sospechan. La astenia constituye el síntoma principal, y la pérdida de peso, debilidad y malestar general pueden ser importantes. En ocasiones las alteraciones de la función gastrointestinal son la primera manifestación ${ }^{4,5}$. La distribución de la hiperpigmentación en codos y areolas son características. El tratamiento se basa en hidrocortisona a dosis de 20-30 $\mathrm{mg} /$ día, duplicando la dosis en situaciones de estrés, cirugía, infecciones, etc.

La HM se produce por una alteración genética infrecuente de carácter autosómico dominante en un $50 \%$ de los casos, en la que los anestésicos inhalados y los relajantes musculares desencadenan una hipertermia progresiva $\left(>45^{\circ} \mathrm{C}\right)$, con rigidez muscular, taquicardia, hipotensión, rabdiomiolisis, y coagulación intravascular diseminada con consecuencias fatales ${ }^{6}$. Este caso revela cómo la elevación mantenida de la CK en un varón joven puede ser la expresión de dos entidades diferentes, como son la EA, en este caso 
una miopatía de origen endocrino, y la condición de portador de una HM, como diagnóstico de sospecha tras la muerte por rabdiomiolisis e hipercaliemia aguda en su hermano. No obstante, no existe disponibilidad en nuestro país para la realización de test genéticos de $\mathrm{HM}$, ni test de contractilidad muscular en biopsia para descartar HM.

\section{BIBLIOGRAFÍA}

1. Maguire AM, Biesheuvel CJ, Ambler GR, Moore B, McLean M, Cowell CT. Evaluation of adrenal function using the human corticotrophin-releasing hormone test, low dose Synacthen test and 9am cortisol level in children and adolescents with central adrenal insufficiency. Clin Endocrinol (Oxf) 2008; 68:683-91.

2. Kong MF, Lawden M, Howlett T. The Addison's disease dilemma-autoimmune or ALD? Lancet 2008; 371:1970.

3. Winqvist $O$, Rorsman F, Kämpe O. Autoimmune adrenal insufficiency: recognition and management. Bio Drugs 2000; 13:107-14.
4. Wiltshire EJ, Wilson R, Pringle KC. Addison's disease presenting with an acute abdomen and complicated by cardiomyopathy. J Paediatr Child Health 2004; 40:644-5.

5. Fernández Serrano F, Collado Sáiz I, Del Cañizo Gómez. Insuficiencia suprarrenal primaria como primera manifestación de un síndrome pluriglandular autoinmune. Semergen 2005; 31:31-4.

6 Litman R, Rosenberg H. Malignant hyperthermia update on susceptibility testing. JAMA 2005; 293:2918-24.

7. Fernández C, Maves de Paula A, Figarella-Branger D, Giorgi R, Chabrol B, Monfort M, et al. Diagnostic evaluation of clinically normal subjects chronic hyperckemia. Neurology 2006; 66:1585-7.

8. Capasso M, De Angelis M, Di Muzio A, Scorciolla O, Pace M, Stuppia L, et al. Familial idiopathic hyper-CK-emia:an underrecognized condition. Muscle Nerve 2006; 33:760-5.

9. Ramírez Espinosa MF, Agulló López JA. A propósito de un caso de eelvación no explicada de la creatincinasa en un varón sano de 20 años de edad. Semergen 2006; 32:243-5.

10. Selva-O'Callaghan A, Labrador-Horrillo M, Gallardo E, Herruzo A, Grau-Junyent JM, Vilardell-Tarres M. Muscle inflammation, autoimmune Addison's disease and sarcoidosis in a patient with dysferlin deficiency. Neuromuscul Disord 2006; 16:208-9. 